\title{
Transverse Vibrations in Beams Supported by a Piece-Wise Homogeneous Visco-Elastic Foundation
}

\author{
Z. Dimitrovová ${ }^{1}$ and L. Frýba ${ }^{2}$ \\ ${ }^{1}$ UNIC, Department of Civil Engineering \\ New University of Lisbon, Portugal \\ ${ }^{2}$ Institute of Theoretical and Applied Mechanics \\ Academy of Sciences of the Czech Republic, Prague, Czech Republic
}

in B.H.V. Topping, L.F. Costa Neves, R.C. Barros, (Editors), "Proceedings of the Twelfth International Conference on Civil, Structural and Environmental Engineering Computing", Civil-Comp Press, Stirlingshire, UK, Paper 44, 2009. doi: $10.4203 /$ ccp. 91.44

\begin{abstract}
Transversal vibrations induced by a load moving uniformly along an infinite beam resting on a piece-wise homogeneous visco-elastic foundation are studied. Special attention is paid to the additional vibrations, conventionally referred to as transition radiations, which arise as the point load traverses the place of foundation discontinuity. The governing equations of the problem are solved by the normalmode analysis. The solution is expressed in a form of infinite sum of orthogonal natural modes multiplied by the generalized coordinate of displacement. The natural frequencies are obtained numerically exploiting the concept of the global dynamic stiffness matrix. This ensures that the frequencies obtained are exact. The methodology has restrictions neither on velocity nor on damping. The approach looks simple, though, the numerical expression of the results is not straightforward. A general procedure for numerical implementation is presented and verified. To illustrate the utility of the methodology parametric optimization is presented and influence of the load mass is studied. The results obtained have direct application in analysis of railway track vibrations induced by high-speed trains when passing regions with significantly different foundation stiffness.
\end{abstract}

Keywords: moving load, moving mass, transversal vibration, transition radiation, normal-mode analysis, dynamic stiffness matrix, natural frequencies, orthonormal mode shapes.

\section{Introduction}

The rapid growth of high-speed railway network and the considerable evolution of train vehicles capable to operate at more than $500 \mathrm{~km} / \mathrm{h}$, gave raise to a number of related problems that have motivated a significant amount of scientific work. The amplification of train-induced vibrations, caused by inhomogeneities in the track foundation, certainly belongs to the issues still demanding further attention. 
Theoretical basis of this occurrence lies in the fact, that when the load passes the place of discontinuity in the supporting structure, additional vibrations, conventionally referred to as transition radiations, are generated. Radiation waves travel backward and forward from the place of discontinuity and may significantly amplify the original waves. As a consequence, the track deterioration is aggravated and concerns related to the vehicle stability and passengers comfort must be taken into account.

The inhomogeneities in the foundation stiffness have origin in geotechnical conditions, in the state of degradation of the track or in an alteration of the structural design. The representative values can differ by orders and their change can be quite sharp. The above mentioned cases refer for instance to situations, where the track settlement due to non-elastic deformations in the ballast and subground is so high, that the full contact between some sleepers and the ballast bed is lost. Then "hanging" sleepers appear and this induces an irregularity of the track stiffness. Other situations cover an embankment-to-bridge or tunnel transition; switch from ballasted to slab track, regions where the high-speed line crosses underground structures, and so on.

An insight into a problem of induced vibrations can be acquired from simplified models, which have a closed form solution. Such approach has many advantages: (i) only main results are available, so they are simple to analyse; (ii) the results preserve parameters dependence allowing for direct sensitivity analysis; (iii) numerical evaluation can be carried out only in places of interest. Due to the simplifying assumptions, however, the results obtained correspond only to the estimate of the real structure response to a moving load.

Simplified one- or two-dimensional models were investigated by other authors. The transition radiation in an infinite string resting on an inhomogeneous elastic foundation, represented by Winkler's model, is studied in [1]. The paper aims to compare the radiation arising due to an abrupt and a smooth change of parameters of the elastic foundation, when a constant load moves uniformly along the string. Problem is solved in the frequency as well as in the time domain. The analysis is restricted to subcritical velocities. In [2], transition radiations in other elastic systems are analysed. The paper developed includes the time domain response of an infinite Euler-Bernoulli beam on an inhomogeneous Winkler foundation subjected to a uniformly moving load. Further development is presented in [3], where instead of the Winkler foundation elastic half-planes are implemented. The analytical solution presented in [2-3] has again limits on load travelling velocity, does not include the effect of damping, the results evaluation is numerically sensitive and, in fact, the methodology is suited for dealing with one discontinuity traversed by a constant force, only. Further analytical study [4] adds a moving mass to the model and assumes periodicity of the inhomogeneous characteristics of the foundation stiffness. The main purpose is to determine conditions under which the vibrations become unstable. 
In this paper, the transient dynamic response of a one-dimensional piece-wise homogeneous structure subjected to uniformly moving time dependent transverse load is expressed in an analytical form exploiting the normal-mode analysis. The structure is assumed in the form of an infinite beam resting on a visco-elastic foundation. The methodology has restrictions neither on velocity magnitude nor on material or mass damping coefficients. Discontinuities in the foundation stiffness parameter, in the flexural rigidity of the beam, in the mass per unit length or in the damping coefficient can be implemented. It is assumed that there is only finite number of discontinuities. Special attention is paid to the transition radiation. The transient shapes of the beam are presented in order to visualize the radiation process.

The concept of the global dynamic stiffness matrix [5] of the structure is used to determine natural frequencies and normal modes. This ensures that the natural frequencies are exact. With this purpose structural elements are defined as the longest possible parts of the structure, where all properties are homogeneous. This is probably the most accurate way to deal with the dynamic behaviour of a distributedparameter beam. Nevertheless, this methodology is not widely used. The reason is the numerical difficulty in evaluation of higher frequencies. Author's previous work [6] focused on avoiding the natural frequencies evaluation and suggested different methodology. Transition radiation was calculated by linking together analytical solutions of each structural element by continuity conditions. In this paper a general procedure for numerical implementation according to the normal-mode analysis is presented and verified. Procedures are programmed in MAPLE [7] and MATLAB [8] environment. To illustrate the utility of the methodology parametric optimization is presented and the influence of the load mass is studied.

The paper is organized in the following way. In Section 2 the motivation for the methodology adopted is given, governing equations are presented and simplifying assumptions are stated. For the sake of simplicity Euler-Bernoulli formulation is presented only. In Section 3 the closed form solution is given and the concept of the global dynamic stiffness matrix is explained. Also the rules for the numerical expression of the results are established and the methodology for extension to infinite beams is presented. Section 4 shows case studies and in Section 5 summary of the achievements and further challenges are stated. Results and conclusions have direct application on knowledge of ground vibrations induced by high-speed trains, especially when the train moves from a region to another one with significantly different foundation stiffness.

\section{Problem statement}

\subsection{Motivation}

An insight into a problem of induced vibrations can be acquired from simplified models, which have a closed form solution. Such approach has many advantages: (i) only main results are available, so they are simple to analyse; (ii) results preserve 
parameters dependence allowing for direct sensitivity analysis; (iii) numerical evaluation can be carried out only in places of interest. Due to the simplifying assumptions, however, the results obtained correspond only to the estimate of the real structure response to a moving load. Nevertheless, it is worthwhile to mention, that the detailed, especially finite element analysis of the track and subgrade structure provides a very large amount of results which are not easy to handle and analyse. Very refined meshes must be used in regions of stiffness discontinuities and the problem must be solved over the whole time domain, which is time consuming. Furthermore, in the standard finite element codes higher natural frequencies are not accurately evaluated, [9]. This error cannot be solved by refining the mesh, because it is inherent to the standard finite element formulation that makes use of cubic Hermite shape functions for the beam elements. The so-called "optical branches" of the discrete spectra diverge with polynomial degree, which shows that higher-order finite elements have no approximability for higher modes in vibration analysis. This shows the fragility of higher-order finite element methods in dynamic applications in which higher modes necessarily participate. Moreover, this error is usually aggravated by the numerical error of the eigenvalues extraction procedure itself.

For this purpose, the commercial general purpose finite element software ANSYS [10] was tested. The error in frequencies of the flexural modes obtained in ANSYS exceeded significantly the error established analytically in [9], which obscured the separation of the so-called acoustic and optical branches in the graph of the error. This can be attributed to the numerical error of the eigenvalues extraction procedure itself. Firstly, simply supported beam of $100 \mathrm{~m}$ length without elastic foundation was modelled. Two standard European rails UIC60 represented the beam. Flexural rigidity and mass per unit length are summarized in Table 3. Block Lanczos extraction method was used to extract first 500 natural frequencies. Results are summarized in Fig. 1. It is seen that the error can reach $29.0 \%$ in 500-th mode (or in the last mode when less elements are used). Next, 100m-length simply supported beam on elastic foundation was tested. It was found that the natural frequencies error is basically independent on the value of the foundation stiffness. Two different models, one of element BEAM54 of the ANSYS library with the capacity of introduction of elastic foundation and the other of BEAM3 with added discrete springs at each node, gave the same results. It was found that the error (for a given beam length) is higher for higher ratio of the flexural rigidity to the mass per unit length of the beam. When the beam was considered as the full track (representative values are around $\mathrm{EI}=66.7 \mathrm{MNm}^{2}, \mu=7200 \mathrm{~kg} / \mathrm{m}$, which attributes high value to $\mathrm{EI} / \mu$ ) the error in 500-th frequency reached already $78.5 \%$.

There is another aggravating factor. First natural frequencies are very similar for relatively strong foundation. This proximity can disorder their sequence. For instance, for foundation stiffness of $10 \mathrm{MN} / \mathrm{m}^{2}$, the first three modes are interchanged in the way that the expected first mode-shape appears in the third position. For foundation stiffness of $40 \mathrm{MN} / \mathrm{m}^{2}$ the first seven (see Fig. 2) and of $100 \mathrm{MN} / \mathrm{m}^{2}$ the first eleven mode shapes are interchanged among themselves. For the previous case of 2UIC60 rails, when foundation stiffness of $100 \mathrm{MN} / \mathrm{m}^{2}$ is 
implemented, then the first five mode shapes are interchanged. In these cases, the total length of $\mathrm{L}=100 \mathrm{~m}$ was discretized into 5000 finite elements.

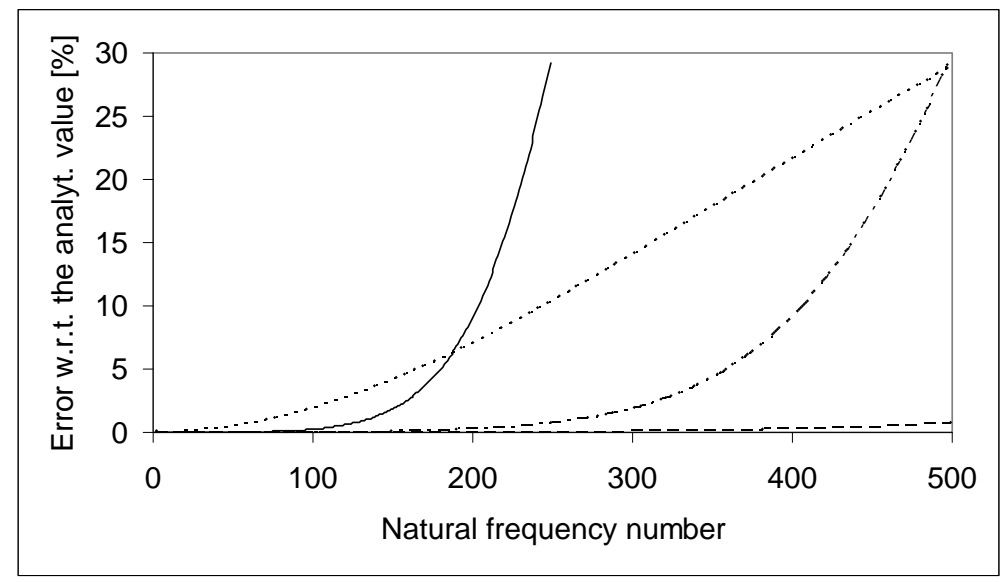

Figure 1: Error in natural frequencies of simply supported beam (250 elements - full line, 500 elements - dot-and-dash line, 1000 elements - dashed line and 5000 elements - dotted line).

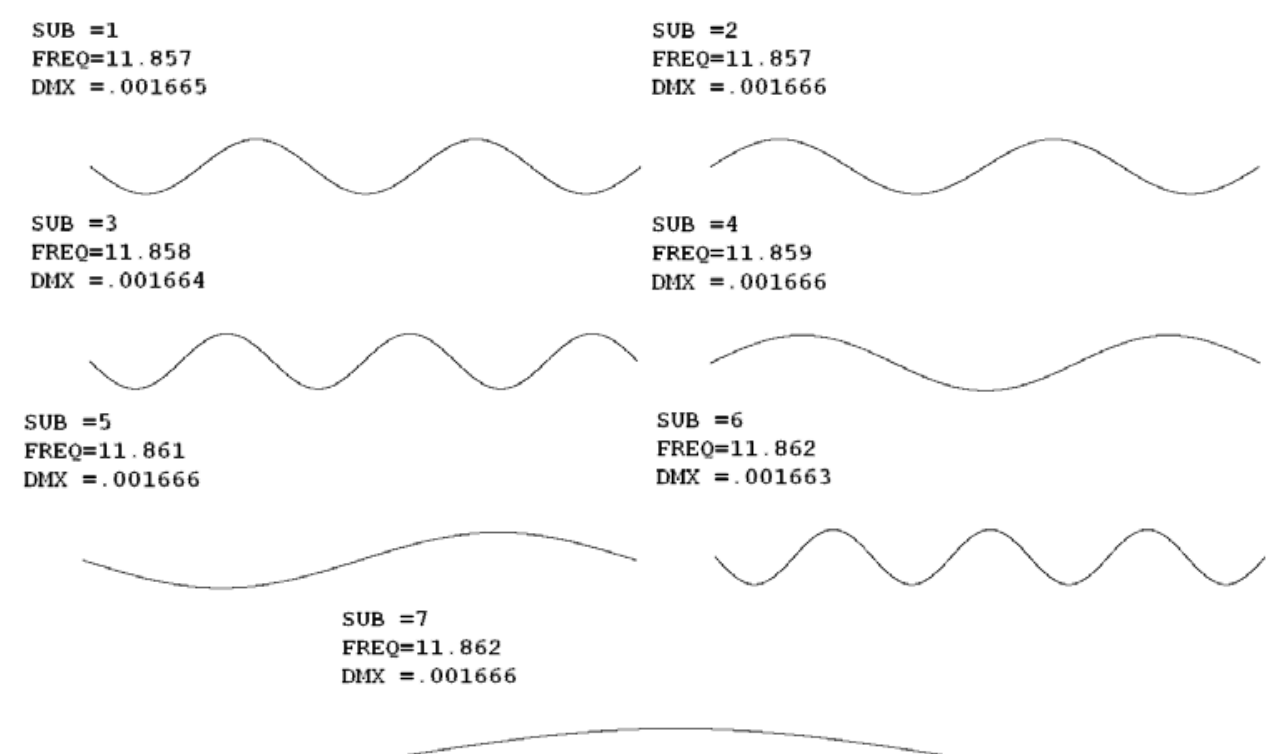

Figure 2: Disorder in natural modes of simply supported beam on elastic foundation, results obtained in ANSYS.

This error affects results obtained by transient analysis with mode superposition solution method implemented. For instance, Figure 3 shows the deflection field related to the force $200 \mathrm{kN}$ travelling by $50 \mathrm{~m} / \mathrm{s}$ on the beam representing the full track, characterized by the previously given values and foundation stiffness of 100 $\mathrm{MN} / \mathrm{m}^{2}$. Deflection field is shown for the force position at $20 \mathrm{~m}$ from the left support and when only first 10 natural modes were used (Figure 3a)) and when 500 natural 
modes were implemented (Figure 3a)). For high number of modes, the results coincide with the full integration method, thus there is no way how to remove in ANSYS the numerical error.
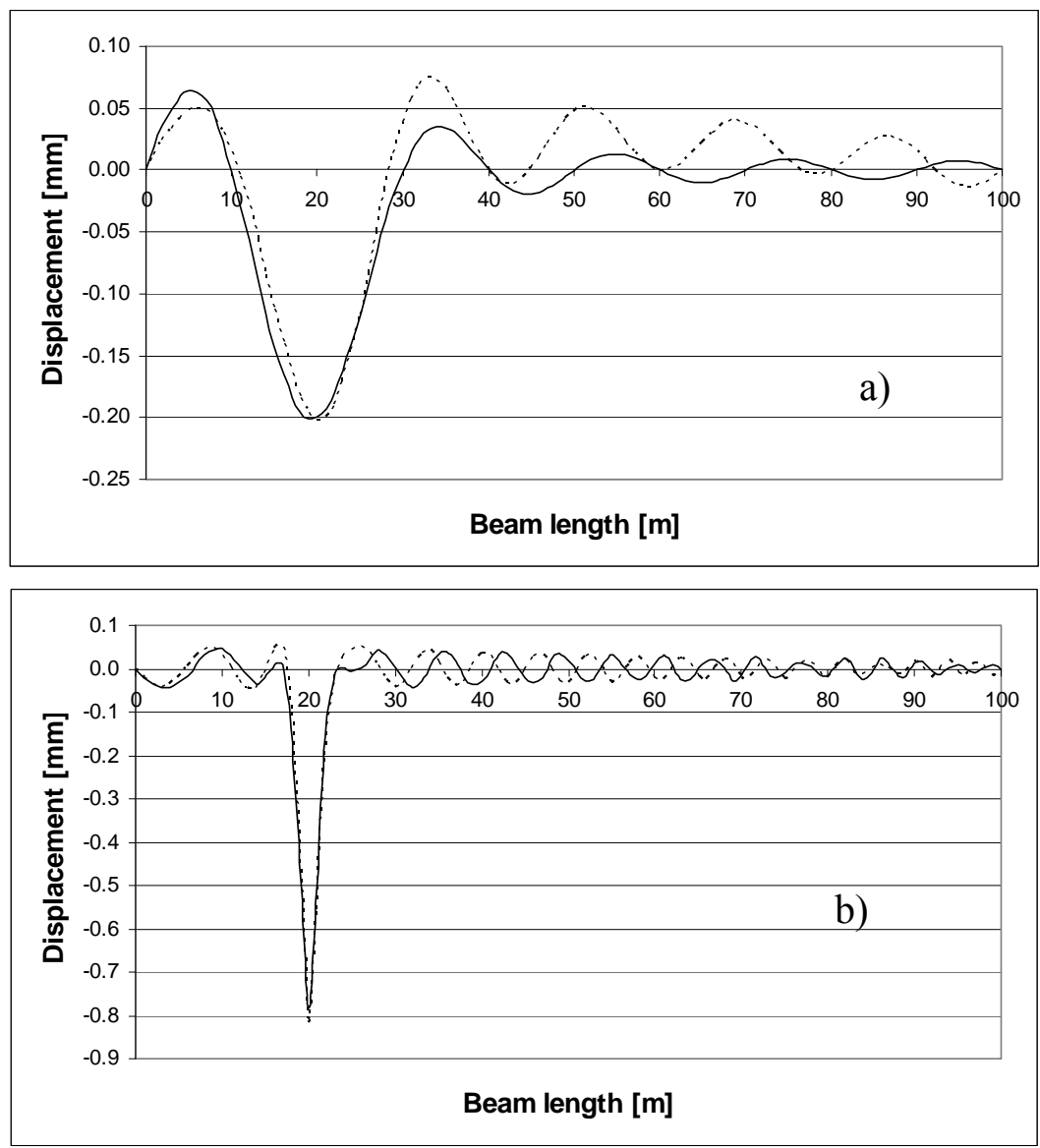

Figure 3: Error in transient solution with mode superposition solution method implemented, ANSYS (dotted curve) and MAPLE (full curve): a) 10 modes implemented, b) 500 modes implemented.

It can therefore be concluded that the results here presented are obtained faster and in a more accurate way, than the ones obtained by standard finite element codes.

\subsection{Governing equations and simplifying assumptions}

Let a uniform motion of a time variable vertical force along a horizontal finite beam on a linear visco-elastic foundation be assumed (Fig. 4). The foundation is modelled as distributed spring-and-dashpot sets. Simplifications for the analysis of vertical vibrations are outlined as follows:

(i) the beam obeys linear elastic Euler-Bernoulli theory;

(ii) the beam damping is proportional to the velocity of vibration. 


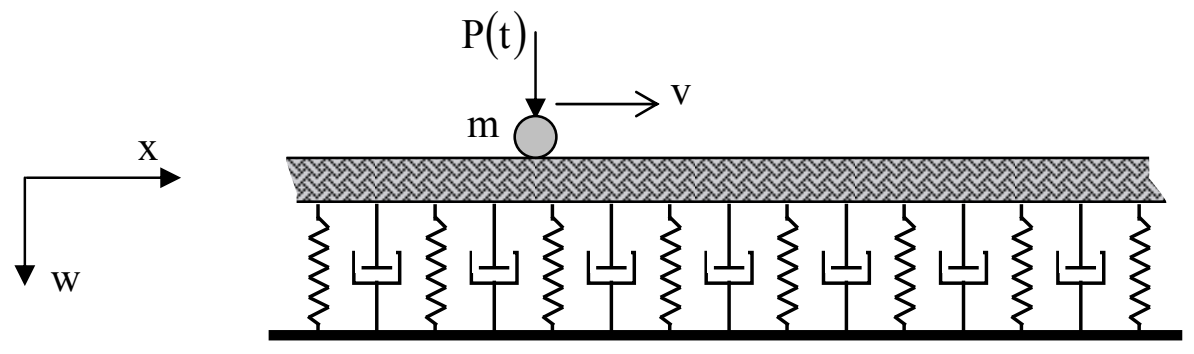

Figure 4: Structure under consideration.

Taking into account the assumptions stated above, the equation of the forced vibration $\mathrm{w}(\mathrm{x}, \mathrm{t})$ reads as [11]:

$$
\begin{array}{r}
\frac{\partial^{2}}{\partial \mathrm{x}^{2}}\left(\mathrm{EI}(\mathrm{x}) \frac{\partial \mathrm{w}^{2}(\mathrm{x}, \mathrm{t})}{\partial \mathrm{x}^{2}}\right)+\mu(\mathrm{x}) \frac{\partial \mathrm{w}^{2}(\mathrm{x}, \mathrm{t})}{\partial \mathrm{t}^{2}}+\mathrm{c}(\mathrm{x}) \frac{\partial \mathrm{w}(\mathrm{x}, \mathrm{t})}{\partial \mathrm{t}}+\mathrm{k}(\mathrm{x}) \mathrm{w}(\mathrm{x}, \mathrm{t})= \\
\delta(\mathrm{x}-\mathrm{vt})\left(\mathrm{P}(\mathrm{t})-\mathrm{m} \frac{\partial \mathrm{w}^{2}(\mathrm{x}, \mathrm{t})}{\partial \mathrm{t}^{2}}\right)
\end{array}
$$

where EI represents the flexural rigidity, $\mu$ the mass per unit length, $\mathrm{c}$ the damping coefficient and $\mathrm{k}$ Winkler constant. $\mathrm{w}$ stands for the transversal displacement, $\mathrm{P}$ for the travelling force and $\mathrm{m}$ for the mass of the load; $\mathrm{w}$ and $\mathrm{P}$ are considered positive when acting downwards. Further in Eq. (1): $\mathrm{v}$ is the constant velocity, $\mathrm{x}$ is the spatial coordinate, $\mathrm{t}$ is the time and $\delta$ is Dirac function. $\mathrm{x}$ has its origin at the left extremity of the structure. Zero time corresponds to force position at $\mathrm{x}=0$. Characteristics EI, $\mu$, $\mathrm{c}$ and $\mathrm{k}$ can have piece-wise constant distribution along the structure. It is assumed that there is only finite number of such discontinuities. Initial and boundary conditions are defined in a standard way. Velocity is maintained constant and no restriction is imposed on its magnitude.

\section{Problem solution}

Eq. (1) can be solved by normal-mode analysis $[5,11]$ :

$$
w(x, t)=\sum_{j=1}^{\infty} q_{j}(t) w_{j}(x)
$$

$\mathrm{q}_{\mathrm{j}}(\mathrm{t})$ are the generalized coordinates of displacement and $\mathrm{w}_{\mathrm{j}}(\mathrm{x})$ are the orthogonal natural modes normalised by:

$$
\mathrm{N}_{\mathrm{j}}=\sqrt{\int_{0}^{\mathrm{L}} \mu(\mathrm{x}) \mathrm{w}_{\mathrm{j}}^{2}(\mathrm{x}) \mathrm{dx}},
$$


where $\mathrm{L}$ it is the total length of the structure.

In order to calculate the undamped natural frequencies and the corresponding normal modes, structure is separated into structural elements with homogeneous properties. Each discontinuity in parameters EI, $\mu, \mathrm{k}$ or $\mathrm{c}$ marks the beginning of a new structural element. Discontinuity in the damping coefficient $\mathrm{c}$ is obviously immaterial for undamped frequencies, however, later on in Eqs. (9) and (11) it will be seen, that this discontinuity must also be considered. Moreover, when the mass of the load is included, another structural node must be added. This one is the most difficult to handle because it varies its position in time. Then the load mass is included in the place of discontinuity as an additional concentrated mass element. On the contrary to the finite elements, only as many as elements, as the number of discontinuities indicates are needed, because the normal modes can be determined exactly within each structural element. In Fig. 5 the degrees of freedom and the member-end generalized harmonic forces are shown.
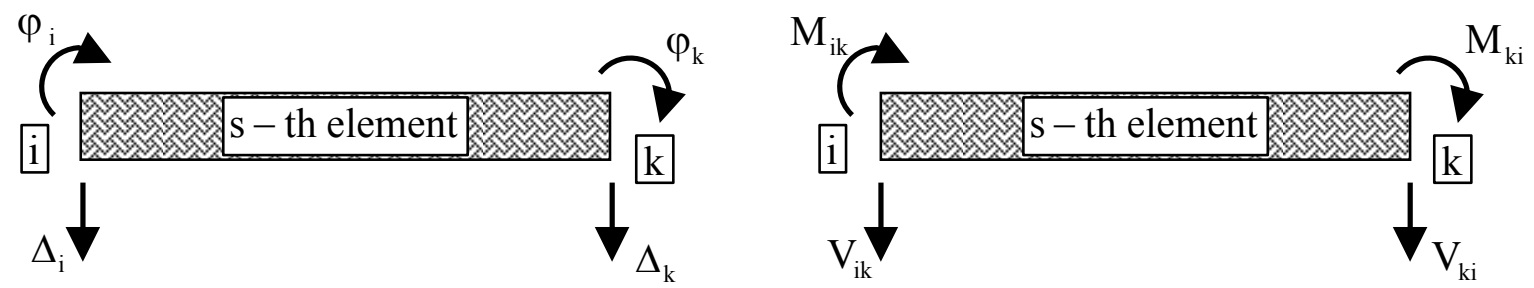

Figure 5: Degrees of freedom and member-end generalized harmonic forces of the sth structural element with nodes $\mathrm{i}$ and $\mathrm{k}$.

Let the full structure be separated into $n$ structural elements. The local dynamic stiffness matrix of clamped-clamped s-th structural element is given in Table 1 (compare with Fig. 2). It is composed by amplitudes of member-end generalized harmonic forces of the element under steady-state vibration exited by harmonic motion of element nodes with a given circular frequency $\omega$. Terms in Table 1 exploit Kolousek's functions [8]:

$$
\begin{gathered}
\mathrm{F}_{1}\left(\lambda_{\mathrm{s}}\right)=-\lambda_{\mathrm{s}} \frac{\sinh \lambda_{\mathrm{s}}-\sin \lambda_{\mathrm{s}}}{\cosh \lambda_{\mathrm{s}} \cos \lambda_{\mathrm{s}}-1}, \mathrm{~F}_{2}\left(\lambda_{\mathrm{s}}\right)=-\lambda_{\mathrm{s}} \frac{\cosh \lambda_{\mathrm{s}} \sin \lambda_{\mathrm{s}}-\sinh \lambda_{\mathrm{s}} \cos \lambda_{\mathrm{s}}}{\cosh \lambda_{\mathrm{s}} \cos \lambda_{\mathrm{s}}-1}, \\
\mathrm{~F}_{3}\left(\lambda_{\mathrm{s}}\right)=-\lambda_{\mathrm{s}}^{2} \frac{\cosh \lambda_{\mathrm{s}}-\cos \lambda_{\mathrm{s}}}{\cosh \lambda_{\mathrm{s}} \cos \lambda_{\mathrm{s}}-1}, \mathrm{~F}_{4}\left(\lambda_{\mathrm{s}}\right)=\lambda_{\mathrm{s}}^{2} \frac{\sinh \lambda_{\mathrm{s}} \sin \lambda_{\mathrm{s}}}{\cosh \lambda_{\mathrm{s}} \cos \lambda_{\mathrm{s}}-1} \\
\mathrm{~F}_{5}\left(\lambda_{\mathrm{s}}\right)=\lambda_{\mathrm{s}}^{3} \frac{\sinh \lambda_{\mathrm{s}}+\sin \lambda_{\mathrm{s}}}{\cosh \lambda_{\mathrm{s}} \cos \lambda_{\mathrm{s}}-1}, \mathrm{~F}_{6}\left(\lambda_{\mathrm{s}}\right)=-\lambda_{\mathrm{s}}^{3} \frac{\cosh \lambda_{\mathrm{s}} \sin \lambda_{\mathrm{s}}+\sinh \lambda_{\mathrm{s}} \cos \lambda_{\mathrm{s}}}{\cosh \lambda_{\mathrm{s}} \cos \lambda_{\mathrm{s}}-1}
\end{gathered}
$$

Parameters $\lambda_{s}$, of different elements are linked together with the excitation frequency as: 


$$
\omega=\sqrt{\frac{\lambda_{1}^{4}}{\mathrm{~L}_{1}^{4}} \frac{\mathrm{EI}_{1}}{\mu_{1}}+\frac{\mathrm{k}_{1}}{\mu_{1}}}=\sqrt{\frac{\lambda_{2}^{4}}{\mathrm{~L}_{2}^{4}} \frac{\mathrm{EI}_{2}}{\mu_{2}}+\frac{\mathrm{k}_{2}}{\mu_{2}}}=\ldots=\sqrt{\frac{\lambda_{\mathrm{n}}^{4}}{\mathrm{~L}_{\mathrm{n}}^{4}} \frac{\mathrm{EI}}{\mu_{\mathrm{n}}}+\frac{\mathrm{k}_{\mathrm{n}}}{\mu_{\mathrm{n}}}} .
$$

\begin{tabular}{|c|c|c|c|c|}
\hline & $\Delta_{\mathrm{i}}$ & $\varphi_{\mathrm{i}}$ & $\Delta_{\mathrm{k}}$ & $\varphi_{\mathrm{k}}$ \\
\hline $\mathrm{V}_{\mathrm{ik}}$ & $\frac{\mathrm{EI}}{\mathrm{L}^{3}} \mathrm{~F}_{6}(\lambda)$ & $-\frac{\mathrm{EI}}{\mathrm{L}^{2}} \mathrm{~F}_{4}(\lambda)$ & $\frac{\mathrm{EI}}{\mathrm{L}^{3}} \mathrm{~F}_{5}(\lambda)$ & $\frac{\mathrm{EI}}{\mathrm{L}^{2}} \mathrm{~F}_{3}(\lambda)$ \\
\hline $\mathrm{M}_{\mathrm{ik}}$ & $-\frac{\mathrm{EI}}{\mathrm{L}^{2}} \mathrm{~F}_{4}(\lambda)$ & $\frac{\mathrm{EI}}{\mathrm{L}} \mathrm{F}_{2}(\lambda)$ & $-\frac{\mathrm{EI}}{\mathrm{L}^{2}} \mathrm{~F}_{3}(\lambda)$ & $\frac{\mathrm{EI}}{\mathrm{L}} \mathrm{F}_{1}(\lambda)$ \\
\hline $\mathrm{V}_{\mathrm{ki}}$ & $\frac{\mathrm{EI}}{\mathrm{L}^{3}} \mathrm{~F}_{5}(\lambda)$ & $-\frac{\mathrm{EI}}{\mathrm{L}^{2}} \mathrm{~F}_{3}(\lambda)$ & $\frac{\mathrm{EI}}{\mathrm{L}^{3}} \mathrm{~F}_{6}(\lambda)$ & $\frac{\mathrm{EI}}{\mathrm{L}^{2}} \mathrm{~F}_{4}(\lambda)$ \\
\hline $\mathrm{M}_{\mathrm{ki}}$ & $\frac{\mathrm{EI}}{\mathrm{L}^{2}} \mathrm{~F}_{3}(\lambda)$ & $\frac{\mathrm{EI}}{\mathrm{L}} \mathrm{F}_{1}(\lambda)$ & $\frac{\mathrm{EI}}{\mathrm{L}^{2}} \mathrm{~F}_{4}(\lambda)$ & $\frac{\mathrm{EI}}{\mathrm{L}} \mathrm{F}_{2}(\lambda)$ \\
\hline
\end{tabular}

Table 1: Amplitudes of member-end generalized harmonic forces of the s-th structural element with nodes $\mathrm{i}$ and $\mathrm{k}$. Subscript s applies to all parameters, but it is omitted for the sake of simplicity.

The global dynamic stiffness matrix is assembled in a standard way by the direct stiffness method and its determinant is set to zero. Roots of this equation, $\omega_{j}$, correspond to the natural frequencies. Substituting $\omega=\omega_{\mathrm{j}}$ back into the global matrix, unknown displacements and rotations at structural nodes can be calculated.

Normal modes are functions composed by well-defined parts within each structural element and linked together by the nodal displacements and rotations. The natural undamped $\mathrm{j}$-th mode of vibration in the s-th structural element reads as:

$$
\begin{aligned}
& \mathrm{w}_{\mathrm{sj}}(\mathrm{x})=\mathrm{A}_{\mathrm{sj}} \sin \frac{\lambda_{\mathrm{sj}}\left(\mathrm{x}-\mathrm{L}_{0 \mathrm{~s}}\right)}{\mathrm{L}_{\mathrm{s}}}+\mathrm{B}_{\mathrm{sj}} \cos \frac{\lambda_{\mathrm{sj}}\left(\mathrm{x}-\mathrm{L}_{0 \mathrm{~s}}\right)}{\mathrm{L}_{\mathrm{s}}} \\
& +\mathrm{C}_{\mathrm{sj}} \sinh \frac{\lambda_{\mathrm{sj}}\left(\mathrm{x}-\mathrm{L}_{0 \mathrm{~s}}\right)}{\mathrm{L}_{\mathrm{s}}}+\mathrm{D}_{\mathrm{sj}} \cosh \frac{\lambda_{\mathrm{sj}}\left(\mathrm{x}-\mathrm{L}_{0 \mathrm{~s}}\right)}{\mathrm{L}_{\mathrm{s}}}, \quad \mathrm{s}=1,2, \ldots \mathrm{n}
\end{aligned}
$$

where $L_{0 s}=\sum_{r=1}^{s-1} L_{r}$ and $L_{r}$ is the length of the $r$-th structural element. Constants from Eq. (6) can be expressed as a function of the nodal displacements and rotations, their forms are given in Table 2.

In order to determine the generalized coordinates of displacement, $q_{j}(t)$, the loading function must also be expanded in series: 


$$
p(x, t)=\sum_{j=1}^{\infty} \mu(x) Q_{j}(t) w_{j}(x) .
$$

\begin{tabular}{|c|c|c|c|c|}
\hline & $\Delta_{\mathrm{i}}$ & $\varphi_{\mathrm{i}}$ & $\Delta_{\mathrm{k}}$ & $\varphi_{\mathrm{k}}$ \\
\hline $\mathrm{A}$ & \multicolumn{4}{|c|}{$\frac{\mathrm{L}}{\lambda} \varphi_{\mathrm{i}}-\mathrm{C}$} \\
\hline $\mathrm{B}$ & $\frac{1}{2 \lambda^{3}} \mathrm{~F}_{6}(\lambda)$ & $-\frac{\mathrm{L}}{2 \lambda^{3}} \mathrm{~F}_{4}(\lambda)+\frac{\mathrm{L}}{2 \lambda}$ & $\frac{1}{2 \lambda^{3}} \mathrm{~F}_{5}(\lambda)$ & $\frac{\mathrm{L}}{2 \lambda^{3}} \mathrm{~F}_{3}(\lambda)$ \\
\hline $\mathrm{C}$ & $\frac{1}{2 \lambda^{2}} \mathrm{~F}_{4}(\lambda)+\frac{1}{2}$ & $-\frac{\mathrm{L}}{2 \lambda^{2}} \mathrm{~F}_{2}(\lambda)$ & $\frac{1}{2 \lambda^{2}} \mathrm{~F}_{3}(\lambda)$ & $-\frac{\mathrm{L}}{2 \lambda^{2}} \mathrm{~F}_{1}(\lambda)$ \\
\hline $\mathrm{D}$ &
\end{tabular}

Table 2: Constants of Eq. (6) for the s-th structural element with nodes i and k. Subscript s applies to all parameters. Within the context of normal modes, subscript $\mathrm{j}$ also applies to all parameters, except of $\mathrm{L}$. Both subscripts are omitted for the sake of simplicity.

Here

$$
Q_{j}(t)=\int_{0}^{L} p(x, t) w_{j}(x) d x
$$

For homogeneous initial conditions, it holds:

$$
q_{j}(t)=\frac{1}{b_{j}(x)} \int_{0}^{t} Q_{j}(\tau) e^{-\frac{c(x)}{2 \mu(x)}(t-\tau)} \sin \left(b_{j}(x)(t-\tau)\right) d \tau,
$$

where

$$
b_{j}(x)=\sqrt{\omega_{j}^{2}-\left(\frac{c(x)}{2 \mu(x)}\right)^{2}}
$$

which for concentrated force (point load) simplifies as:

$$
\mathrm{q}_{\mathrm{j}}(\mathrm{t})=\frac{1}{\mathrm{~b}_{\mathrm{j}}(\mathrm{x})} \int_{0}^{\mathrm{t}} \mathrm{P}(\tau) \mathrm{w}_{\mathrm{j}}(\mathrm{v} \tau) \mathrm{e}^{-\frac{\mathrm{c}(\mathrm{x})}{2 \mu(\mathrm{x})}(\mathrm{t}-\tau)} \sin \left(\mathrm{b}_{\mathrm{j}}(\mathrm{x})(\mathrm{t}-\tau)\right) \mathrm{d} \tau
$$

If the load mass is included, Eq. (11) cannot be used anymore, because the integration over $\mathrm{w}$ cannot be performed as its form varies in time. In this case one has to go back to Eq. (9). In fact it is possible to assume, that function Q varies 
linearly over each time interval $\left[\mathrm{t}_{\mathrm{i}-1}, \mathrm{t}_{\mathrm{i}}\right]$, where $\Delta \mathrm{t}=\mathrm{t}_{\mathrm{i}}-\mathrm{t}_{\mathrm{i}-1}$ can be assumed constant, but it must be sufficiently small. Then an intermediate value within such interval reads as:

$$
\mathrm{Q}_{j}(\bar{\tau})=\mathrm{Q}_{\mathrm{j}}\left(\mathrm{t}_{\mathrm{i}-1}\right)+\frac{\mathrm{Q}_{\mathrm{j}}\left(\mathrm{t}_{\mathrm{i}}\right)-\mathrm{Q}_{\mathrm{j}}\left(\mathrm{t}_{\mathrm{i}-1}\right)}{\Delta \mathrm{t}} \bar{\tau},
$$

where $\bar{\tau}$ is a local time starting at $t_{i-1}$. After some manipulations (in the following relations $\mathrm{x}$-dependence is omitted for the sake of simplicity, value corresponding to the actual time must be used):

$$
\begin{gathered}
\mathrm{q}_{\mathrm{j}}\left(\mathrm{t}_{\mathrm{i}}\right)=\mathrm{e}^{-\frac{\mathrm{c}}{2 \mu} \Delta t}\left(\mathrm{D}_{\mathrm{i}} \cos \left(\mathrm{b}_{\mathrm{i}, \mathrm{j}} \Delta \mathrm{t}\right)+\mathrm{E}_{\mathrm{i}} \sin \left(\mathrm{b}_{\mathrm{i}, \mathrm{j}} \Delta \mathrm{t}\right)\right)+\frac{\mathrm{d}_{\mathrm{i}}}{\mathrm{b}_{\mathrm{i}, \mathrm{j}}^{2}}+\frac{\mathrm{e}_{\mathrm{i}} \Delta \mathrm{t}}{\mathrm{b}_{\mathrm{i}, \mathrm{j}}^{2}}, \\
\frac{\mathrm{d}}{\mathrm{dt}} \mathrm{q}_{\mathrm{j}}\left(\mathrm{t}_{\mathrm{i}}\right)=-\frac{\mathrm{c}}{2 \mu}\left(\mathrm{q}_{\mathrm{j}}\left(\mathrm{t}_{\mathrm{i}}\right)-\frac{\mathrm{d}_{\mathrm{i}}}{\mathrm{b}_{\mathrm{i}, \mathrm{j}}^{2}}--\frac{\mathrm{e}_{\mathrm{i}} \Delta \mathrm{t}}{\mathrm{b}_{\mathrm{i}, \mathrm{j}}^{2}}\right) \\
+\mathrm{b}_{\mathrm{i}, \mathrm{j}} \mathrm{e}^{-\frac{\mathrm{c}}{2 \mu}}\left(-\mathrm{D}_{\mathrm{i}} \sin \left(\mathrm{b}_{\mathrm{i}, \mathrm{j}} \Delta \mathrm{t}\right)+\mathrm{E}_{\mathrm{i}} \cos \left(\mathrm{b}_{\mathrm{i}, \mathrm{j}} \Delta \mathrm{t}\right)\right)+\frac{\mathrm{e}_{\mathrm{i}}}{\mathrm{b}_{\mathrm{i}, \mathrm{j}}^{2}}
\end{gathered}
$$

where

$$
\begin{gathered}
d_{i}=Q_{j}\left(t_{i-1}\right), e_{i}=\frac{Q_{j}\left(t_{i}\right)-Q_{j}\left(t_{i-1}\right)}{\Delta t}, D_{i}=q_{j}\left(t_{i-1}\right)-\frac{d_{i}}{b_{i, j}^{2}}, \\
E_{i}=\frac{q_{j}\left(t_{i-1}\right) \frac{c}{2 \mu}+\frac{d}{d t} q_{j}\left(t_{i-1}\right)}{b_{i, j}}-\frac{e_{i}}{b_{i, j}^{3}} .
\end{gathered}
$$

Expressions presented in this section are rather simple; the difficulties arise in numerical expression of the results. Determination of natural frequencies is considered the most difficult part of the numerical assessment of the results expressed by Eq. (2). This difficulty is aggravated by the fact, that very high number of natural frequencies is required in problems with foundation discontinuity. After the global matrix has been assemblaged, Eq. (5) can be substituted, and the determinant can be expressed in terms of a single unknown, $\omega$. Actually, it is more convenient to express the determinant in terms of $\omega^{2}$ and search for $\omega^{2}$ instead of $\omega$. Only numerical methods, can be used for roots search. The determinant has many singularities coincident with all natural frequencies of each structural element considered separately. The denominator consists of product of expressions: $H_{1}\left(\lambda_{1}\right)$, $\cosh \lambda_{\mathrm{s}} \cos \lambda_{\mathrm{s}}-1, \mathrm{~s}=2 . . \mathrm{n}-1$ and $\mathrm{H}_{\mathrm{n}}\left(\lambda_{\mathrm{n}}\right)$, which correspond to the left hand side of the characteristic equation of each member. The middle relations refer to the clamped-clamped beam. First and last expression can be different, depending on the actual boundary conditions at the extremities of the full structure. In order to avoid 
special treatment around the singularities, it is possible to solve the roots only in the nominator. Then software which can handle very large numbers and introduce highdigits precision must be used. Only in MAPLE (or similar symbolical calculation software) it is possible to: (a) increase digits precision without any link to the computed specified value; (b) deal with very high numbers. Therefore most of the procedures of numerical evaluation of the results were programmed in MAPLE environment, some of them could be placed also in MATLAB.

The methodology for extension to infinite beams by mitigation the boundary conditions effect is defined in the following way. It is assumed that the force starts to actuate at a length $\mathrm{L}_{\mathrm{i}}$ from the left support and that it varies from zero to its final value over an intermediate region $\mathrm{L}_{\mathrm{c}}$. The time variation of the force is assumed in a sinusoidal shape in order to keep the time derivatives continues. In more details:

$$
P(t)= \begin{cases}0 & \text { for } t \leq \frac{L_{i}}{v} \\ \frac{P_{0}}{2}\left(1+\sin \left(\pi\left(\frac{v t}{L_{c}}-\frac{L_{i}}{L_{c}}-\frac{1}{2}\right)\right)\right) & \text { for } \frac{L_{i}}{v}<t \leq \frac{L_{i}+L_{c}}{v} \\ P_{0} & \text { for } t>\frac{L_{i}+L_{c}}{v}\end{cases}
$$

where $\mathrm{P}_{0}$ is the final value of the force applied. This methodology ensures that the maximum displacement smoothly increases till its final value, which corresponds to the analytical maximum of the quasi-stationary regime. It would be possible to assume only linear variation of the force applied. Nevertheless, it was verified that if the time derivatives of the variation function are continues, it is better prevented possible oscillation of the final value. The question is how long $L_{i}$ and especially $L_{c}$ should be. It is suggested and verified that analogy with a representative spring can be used. For the sake of simplicity it is assumed that the force applied increases linearly. Thus, let a ramped force with the maximum value $\mathrm{F}$ be applied on a mass $\mathrm{m}$ supported by a spring of the rigidity $\mathrm{K}$. Time variation of the mass displacement can be expressed analytically by:

$$
\mathrm{u}(\mathrm{t})=\frac{\mathrm{F}}{\mathrm{K}}+\frac{\mathrm{F} \sqrt{\mathrm{m}}}{\mathrm{t}_{\mathrm{c}} \sqrt{\mathrm{K}^{3}}}\left(\sin \left(\sqrt{\frac{\mathrm{K}}{\mathrm{m}}}\left(\mathrm{t}-\mathrm{t}_{\mathrm{c}}\right)\right)-\sin \left(\sqrt{\frac{\mathrm{K}}{\mathrm{m}} \mathrm{t}}\right)\right),
$$

where $t_{c}$ is the time over which the force increases linearly. The second term on the right hand side of Eq. (15) stands for the oscillating part of the solution. The amplitude of this oscillation is: 


$$
\frac{\mathrm{F}}{\mathrm{t}_{\mathrm{c}}} \sqrt{\frac{2 \mathrm{~m}\left(1-\cos \left(\sqrt{\left.\left.\frac{\mathrm{K}}{\mathrm{m}} \mathrm{t}_{\mathrm{c}}\right)\right)}\right.\right.}{\mathrm{K}^{3}}} .
$$

By implementation lower time $t_{c}$, the amplitude gets generally higher, but there is an additional oscillation in the amplitude itself. For our purpose, it is enough to choose the time $t_{c}$, in a way to force the amplitude to a "reasonable" part of $F / K$. This requirement, as expected, does not depend on the force applied, but it does depend on $\mathrm{m}$ and $\mathrm{K}$. In accordance with the analogy, $\mathrm{K}$ will be replaced by $\mathrm{k}$ and $\mathrm{m}$ by $\mu$.

\section{Case studies}

\subsection{Parametric optimization}

In this case study two UIC60 rails are used to model the beam. The load applied is approximated by the total axle mass of $17000 \mathrm{~kg}$ corresponding to a locomotive of the Thalys high-speed train. A good isolation device and ideal rail surface is assumed, so that the harmonic component can be neglected and the load can be modelled as a constant moving force. All numerical input data are summarized in Table 3 .

\begin{tabular}{|c|c|}
\hline Property & $\begin{array}{c}\text { Beam } \\
(2 \text { rails UIC60) }\end{array}$ \\
\hline Young's modulus E $(\mathrm{GPa})$ & 210 \\
\hline Moment of inertia $\mathrm{I}\left(\mathrm{m}^{4}\right)$ & $6110 \cdot 10^{-8}$ \\
\hline Density per unit length $\mu(\mathrm{kg} / \mathrm{m})$ & 119.87 \\
\hline Maximum force applied $\mathrm{P}_{0}(\mathrm{kN})$ & 166.8 \\
\hline
\end{tabular}

Table 3: Numerical input data used in examples.

The probability that an admissible upward displacement will be exceeded, when the load passes by a certain velocity obeying the normal distribution, is determined. For this optimization problem it is assumed that the given structure has an intermediate part of adaptable foundation stiffness. The length of this intermediate part is fixed, and its foundation stiffness is optimized. In the study shown below, Winkler constant in the soft region is implemented as $1000 \mathrm{kN} / \mathrm{m}^{2}$, in the strong one is 10 times higher, i.e. $10000 \mathrm{kN} / \mathrm{m}^{2}$, and the intermediate region length is chosen as $6 \mathrm{~m}$, covering therefore 10 sleepers. Lengths of the soft and of the strong parts, their separation, number of implemented modes and other numerical data are the same as in the previous study. Reference velocity is chosen as $150 \mathrm{~m} / \mathrm{s}$, obeying normal distribution with standard deviation of $3 \mathrm{~m} / \mathrm{s}$. Passage of the load in both directions is investigated. 
Analysis is performed in a parametric way with respect to the velocity and with respect to the intermediate foundation stiffness. During the analysis the velocity is varied by $10 \mathrm{~m} / \mathrm{s}$ from 140 to 160 , to accommodate the chosen standard deviation. The maximum upward displacement was selected as the most harmful effect in this analysis. Evolution of this displacement with the load position is shown in Fig. 6 in the structure without an intermediate length. Regarding the passage forward, results of optimization show, that very small increase in foundation stiffness with respect to the soft value will attenuate the deflection wave propagation in the soft region and, at the same time, will ensure that the displacement shape is affected in the way that the next discontinuity in foundation stiffness does not cause the large peak in the response (Figs. 7 and 8).

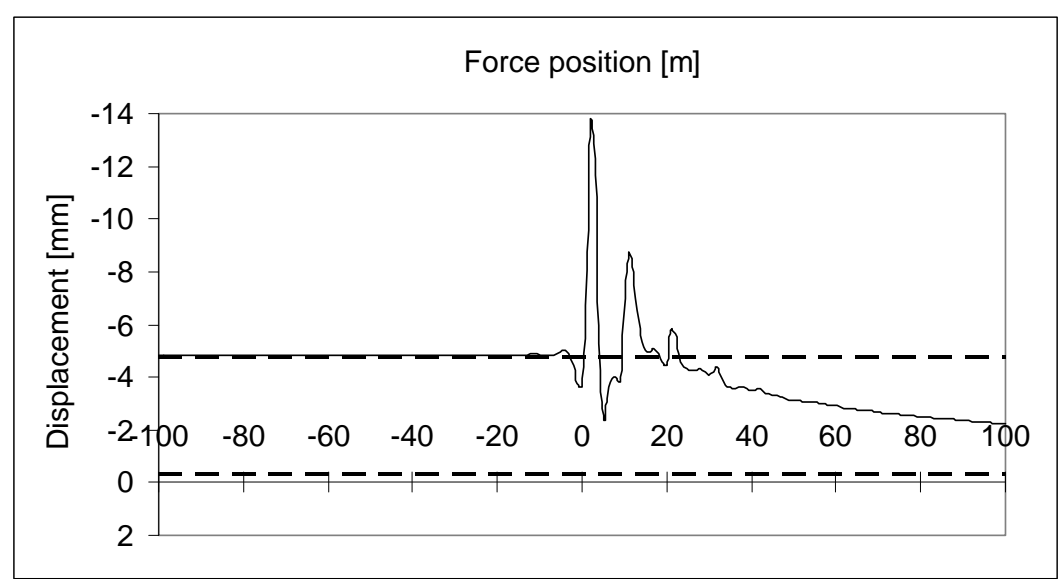

Figure 6: Maximum upward displacement, passage forward by velocity $\mathrm{v}=150 \mathrm{~m} / \mathrm{s}$, dashed lines are used for the quasi-stationary solutions.

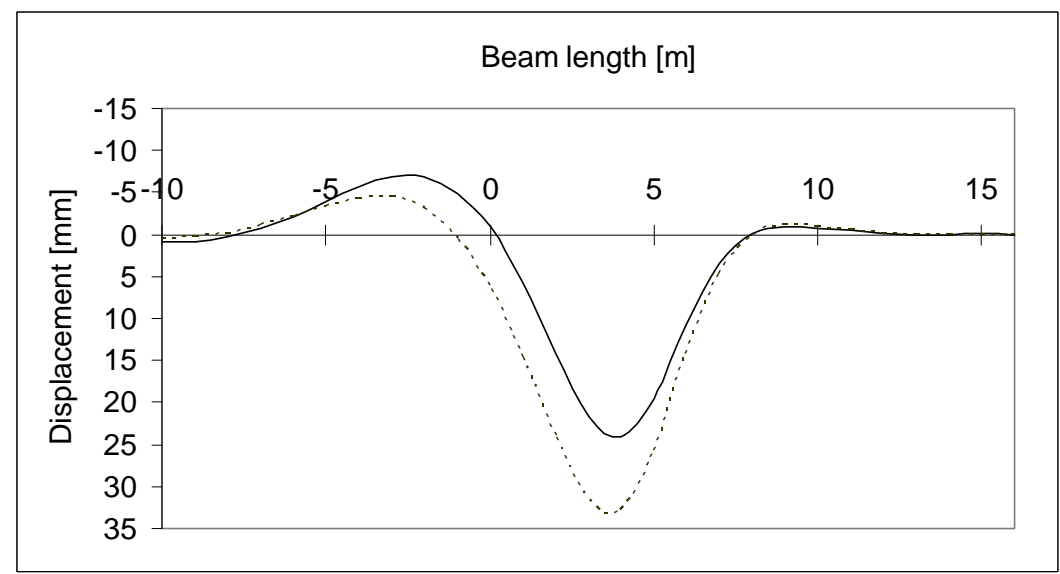

Figure 7: Displacement from the optimized solution (full curve) compared with the solution without the intermediate region (dotted curve), load position where the maximum in the optimized solution is achieved (load position at $4 \mathrm{~m}$ ). 


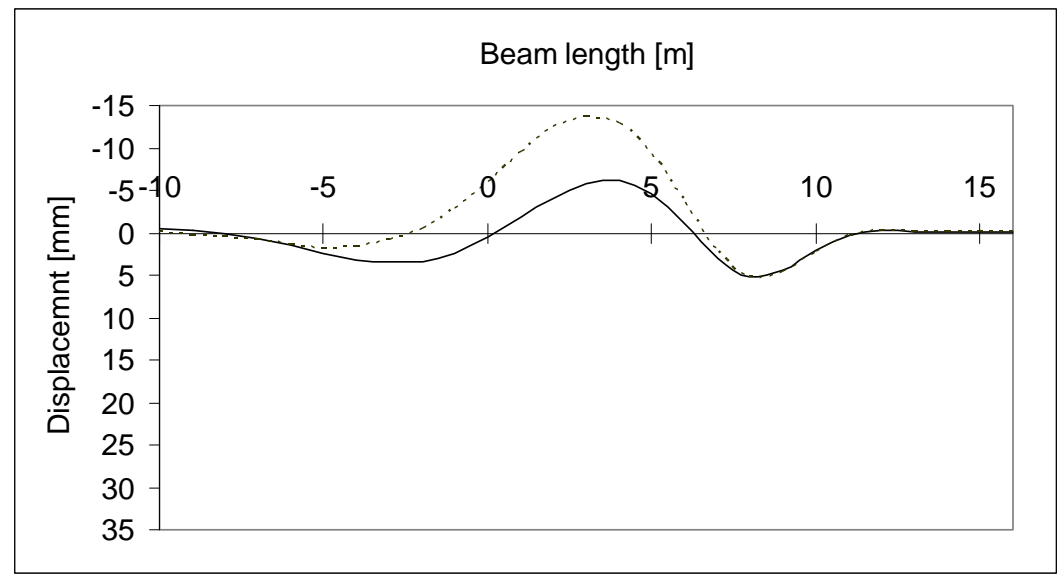

Figure 8: Displacement from the optimized solution (full curve) compared with the solution without the intermediate region (dotted curve), load position where the maximum in the non-optimized solution is achieved (load position at $8 \mathrm{~m}$ ).

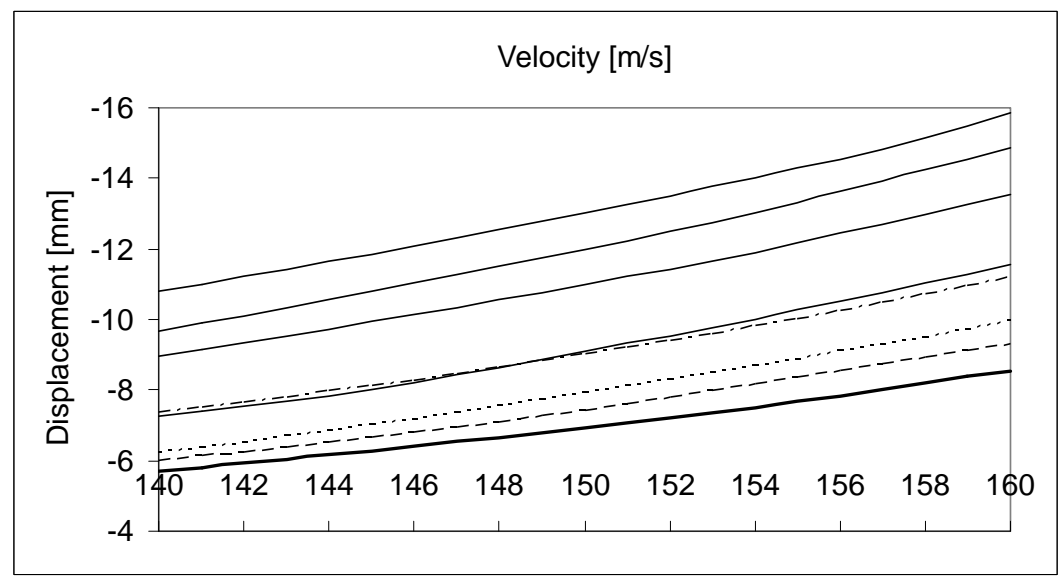

Figure 9: Maximum upward displacement as a function of velocity, passage forward. Bold curve shows the optimum solution $\left(1400 \mathrm{kN} / \mathrm{m}^{2}\right)$, the closest values are for $1500 \mathrm{kN} / \mathrm{m}^{2}$ (dashed), $1600 \mathrm{kN} / \mathrm{m}^{2}$ (dotted), $1200 \mathrm{kN} / \mathrm{m}^{2}$ (dot-and-dash line), other four full curves refer to $2000 \mathrm{kN} / \mathrm{m}^{2}, 3000 \mathrm{kN} / \mathrm{m}^{2}, 4000 \mathrm{kN} / \mathrm{m}^{2}, 8000 \mathrm{kN} / \mathrm{m}^{2}$ (the highest).

Reduction by $50 \%$ was obtained by the optimization procedure. Similar features were verified for the opposite load movement (passage backward), however, now only the reduction by $28 \%$ was achieved. Figs. 9 and 10 show the maximum displacement as a function of the velocity for the forward and the backward passage. It is seen that the stiffness of $1400 \mathrm{kN} / \mathrm{m}^{2}$ is the optimum value for the passage forward and $1500 \mathrm{kN} / \mathrm{m}^{2}$ for the passage backward. Displacement values are compared in Table 4. In Figs. 11 and 12 the deflection curves reaching the maximum are recorded, which indicate the load position in such cases. Each curve corresponds to a different velocity. In passage forward the maximum is always achieved at the same load position. This is not verified for the passage backward. Results are affected by the step chosen for the numerical evaluation $1 \mathrm{~m}$. 


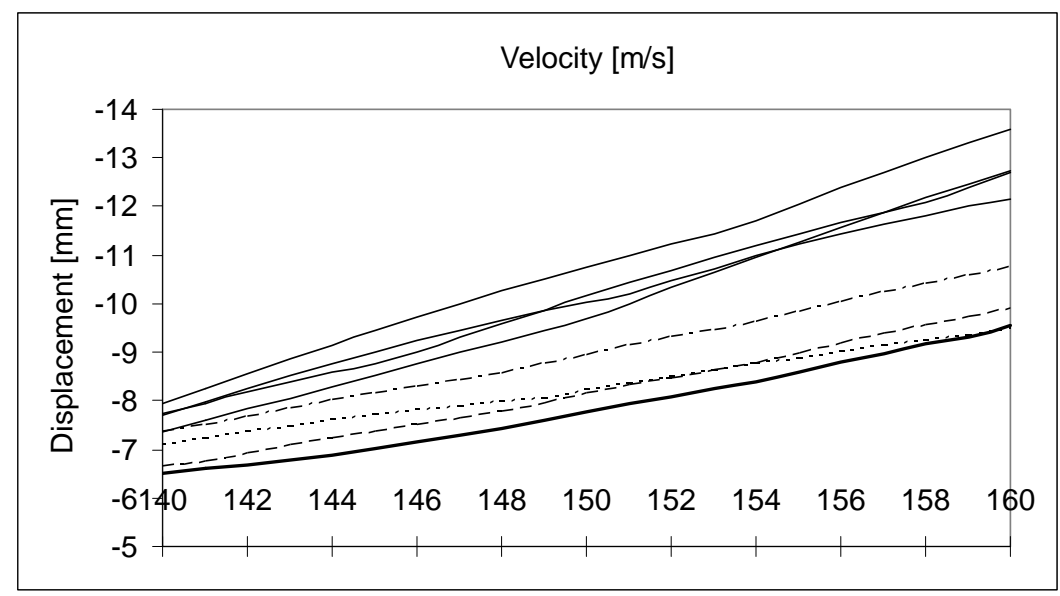

Figure 10: Maximum upward displacement as a function of velocity, passage backward. Bold curve shows the optimum solution $\left(1500 \mathrm{kN} \mathrm{m}^{-2}\right)$, the closest values are for $1400 \mathrm{kN} \mathrm{m}^{-2}$ (dashed), $1600 \mathrm{kN} \mathrm{m}^{-2}$ (dotted), $1200 \mathrm{kN} \mathrm{m}^{-2}$ (dot-and-dash line), other four full curves refer to $2000 \mathrm{kN} \mathrm{m}^{-2}, 3000 \mathrm{kN} \mathrm{m}^{-2}, 4000 \mathrm{kN} \mathrm{m}^{-2}, 8000$ $\mathrm{kN} \mathrm{m}^{-2}$ (the highest).

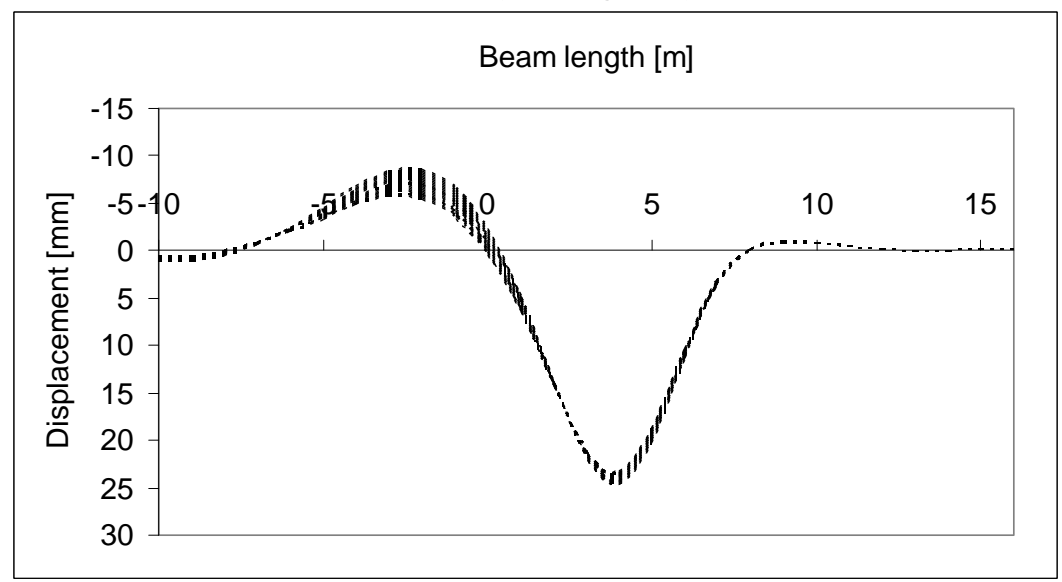

Figure 11: Maximum upward displacement curves as a function of velocity, passage forward. Zero position is at the first discontinuity.

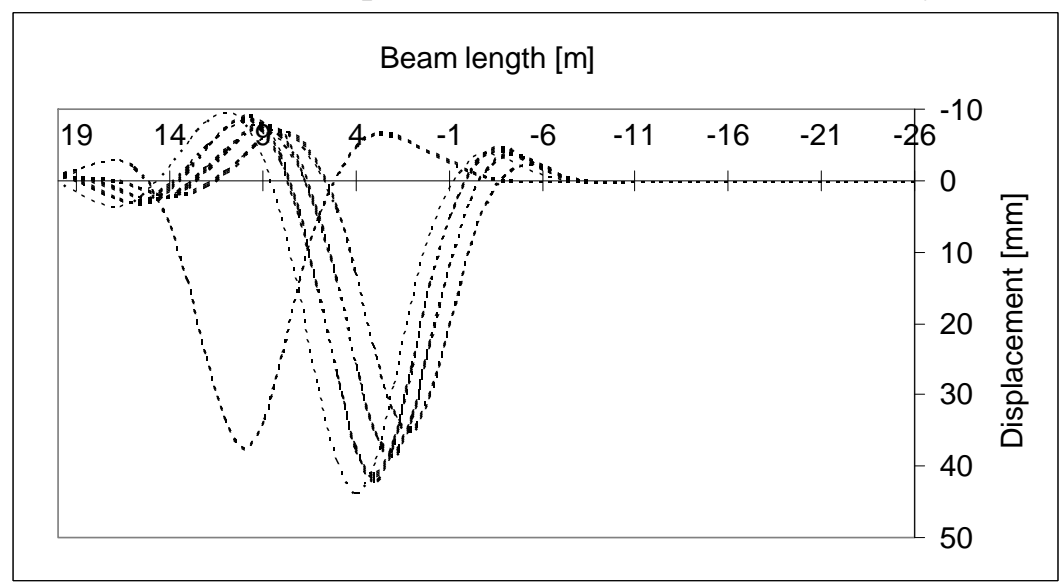

Figure 12: Maximum upward displacement curves as a function of velocity, passage backward. Zero position is at the second discontinuity. 
The main advantage of this analysis is the fact, that the analytical solution is used, therefore in each studied case only a small region before and after the intermediate region can be analysed to find the maximum. 10m-length is enough for the passage forward, slightly more is necessary for the passage backward (20m-length was used).

\begin{tabular}{|c|c|c|}
\hline $\begin{array}{c}\text { Maximum upward } \\
\text { displacement [mm }]\end{array}$ & $\begin{array}{c}\text { Passage forward } \\
\left(150 \mathrm{~m} \mathrm{~s}^{-1}\right)\end{array}$ & $\begin{array}{c}\text { Passage backward } \\
\left(150 \mathrm{~m} \mathrm{~s}^{-1}\right)\end{array}$ \\
\hline No intermediate part & 13.72 & 10.83 \\
\hline $\begin{array}{c}\text { Intermediate stiffness } \\
1400 \mathrm{kN} \mathrm{m}^{-2}\end{array}$ & 6.92 & 8.13 \\
\hline $\begin{array}{c}\text { Intermediate stiffness } \\
1500 \mathrm{kN} \mathrm{m}^{-2}\end{array}$ & 7.42 & 7.77 \\
\hline
\end{tabular}

Table 4: Maximum upward displacement for the passage by the reference velocity.

As a positive conclusion of this analysis is the fact, that the optimum intermediate stiffness for the forward and the backward passage are very similar, which is an important conclusion for practical use. In order to complete the optimization analysis, admissible values of 7 and $8 \mathrm{~mm}$ for the upward displacement were chosen. The probability of their exceeding is summarized in Table 5. It can thus be concluded that the optimum intermediate stiffness in this problem is $1400 \mathrm{kN} / \mathrm{m}^{2}$ and the probability of exceeding the admissible displacements of 7 and $8 \mathrm{~mm}$ is $70.7 \%$ and $30.4 \%$, respectively.

\begin{tabular}{|c|c|c|c|c|c|c|}
\hline \multirow{2}{*}{ Probability } & \multicolumn{3}{|c|}{ Admissible value $8 \mathrm{~mm}$} & \multicolumn{3}{c|}{ Admissible value 7mm } \\
\cline { 2 - 7 } & $\begin{array}{c}\text { Passage } \\
\text { forward }\end{array}$ & $\begin{array}{c}\text { Passage } \\
\text { backward }\end{array}$ & Total & $\begin{array}{c}\text { Passage } \\
\text { forward }\end{array}$ & $\begin{array}{c}\text { Passage } \\
\text { backward }\end{array}$ & Total \\
\hline $\begin{array}{c}\text { Intermediate } \\
\text { stiffness } \\
1400 \mathrm{kN} / \mathrm{m}^{2}\end{array}$ & $1.0 \%$ & $59.7 \%$ & $30.4 \%$ & $42.2 \%$ & $99.3 \%$ & $70.7 \%$ \\
\hline $\begin{array}{c}\text { Intermediate } \\
\text { stiffness } \\
1500 \mathrm{kN} / \mathrm{m}^{2}\end{array}$ & $14.0 \%$ & $31.9 \%$ & $23.0 \%$ & $79.9 \%$ & $95.7 \%$ & $87.8 \%$ \\
\hline
\end{tabular}

Table 5: Probabilities of exceeding the admissible values.

\subsection{Effect of the mass of the load}

In this case study first of all benchmark problem was solved by the methodology presented in Section 3. The beam was modelled as left cantilever of $\mathrm{L}=7.62 \mathrm{~m}$, $\mathrm{EI}=9.47 \mathrm{MNm}^{2}, \mu=46 \mathrm{~kg} / \mathrm{m}$. The moving mass had $\mathrm{m}=2579 \mathrm{~kg}$ with the corresponding weigth of $25.3 \mathrm{kN}$. The velocity applied was $50.8 \mathrm{~m} / \mathrm{s}$. In Fig. 13 the displacement at the free extremity of the beam and in Fig. 14 the deflection curve corresponding to an intermediate position of the mass at $3.8 \mathrm{~m}$ are shown. 


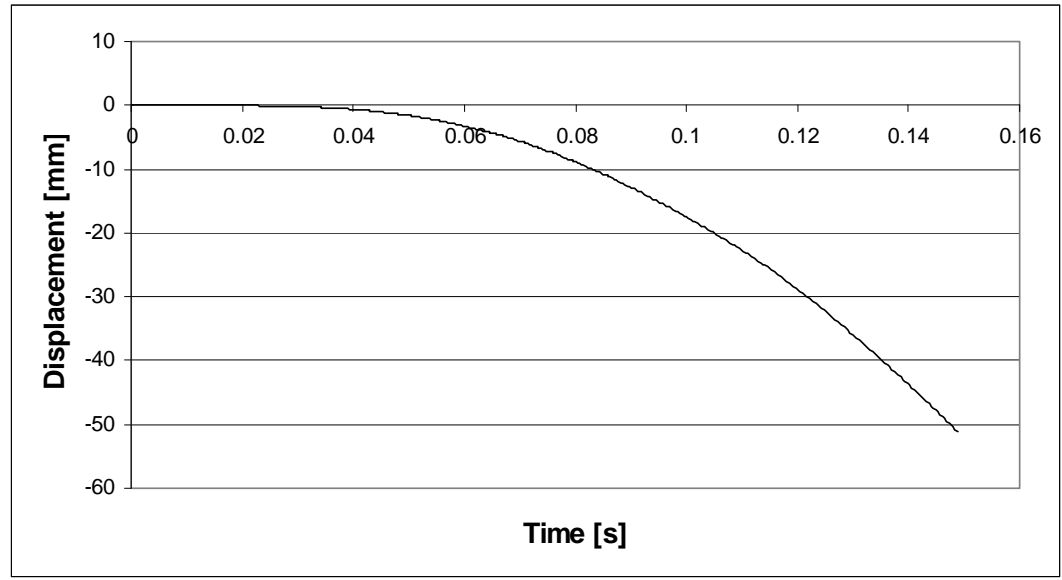

Figure 13: Displacement at the free extremity of the beam with respect to time.

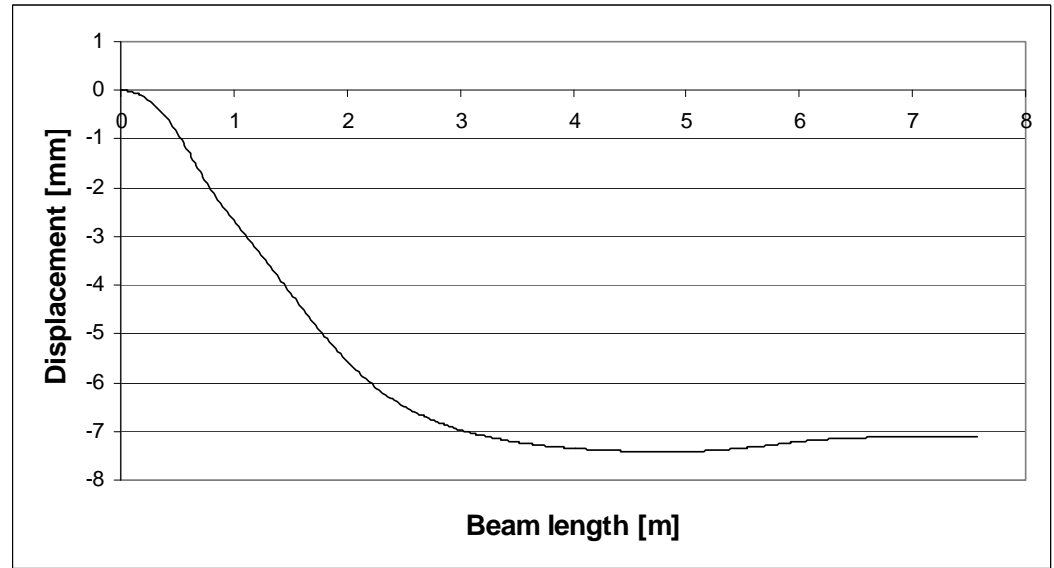

Figure 14: Deflection curve for the mass position at $3.8 \mathrm{~m}$.

Results presented exactly match the expected ones presented in [12].

\section{Conclusions and further development}

The transversal vibrations induced by a load moving uniformly along an infinite beam resting on a piece-wise homogeneous visco-elastic foundation has been investigated. Special attention has been paid to the additional vibrations, conventionally referred to as transition radiations, which arise as the point load traverses the place of the foundation discontinuity. The governing equations of the problem have been solved by the normal-mode analysis. Procedures were programmed in MAPLE and MATLAB environment. Displacement field is presented in a closed form as an analytical result, therefore numerical evaluation can be carried out only in places of interest. Results presented show high level of accuracy, because they do not contain the error of the standard finite element method and the calculated natural frequencies and shapes are exact. 
The methodology presented has restrictions neither on velocity magnitude nor on damping. Load can be represented by a set of moving forces which magnitudes can be time dependent. Effect of the mass of the load can be included. Then normal modes must be evaluated at each time step and some of the previously mentioned advantages are lost.

Although focusing only on one-dimensional systems under physical and geometrical linearity this study provides an important insight into the problem of excessive ground vibrations induced by high-speed trains passing in sections where an abrupt change in vertical stiffness occurs. The methodology has some additional potentialities that will be explored in the future work. Among them it might be mentioned implementation of more complicated structural elements like an elevated railway, which can be modelled as a layered-beam system that is composed of two parallel beams with visco-elastic layer in between.

The results obtained have direct application in the analysis of railway track vibrations induced by high-speed trains when passing regions with significantly different foundation stiffness. The conclusions of this paper might be served as a design basis for the treatment of solution for such regions.

\section{Acknowledgements}

The first author would like to thank to Fundação para a Ciência e a Tecnologia, of the Portuguese Ministry of Science and Technology for supporting expenses related to this congress (grant PTDC/EME-PME/67658/2006: "Design of cellular elastomeric materials for passive vibration control"). The second author appreciates the support of the grant agency of the Czech Republic GA CR 103/08/1340.

\section{References}

[1] A.V. Metrikine, A.R.M. Wolfert, H.A. Dieterman, "Transition radiation in an elastically supported string. Abrupt and smooth variations of the support stiffness", Wave Motion 27, 291-305, 1998.

[2] K.N. Van Dalen, Ground Vibration Induced by a High-Speed Train Running over Inhomogeneous Subsoil, Transition Radiation in Two-Dimensional Inhomogeneous Elastic Systems, Master Thesis, Department of Structural Engineering, TUDelft, 2006.

[3] K.N. van Dalen, A.V. Metrikine, "Transition radiation of elastic waves at the interface of two elastic half-planes", Journal of Sound and Vibration 310, 702 717, 2008.

[4] S.N. Verichev, A.V. Metrikine, "Instability of vibrations of a mass that moves uniformly along a beam on a periodically inhomogeneous foundation", Journal of Sound and Vibration 260, 901-925, 2003.

[5] V. Koloušek, "Dynamics in Engineering Structures". Academia, Prague, Butterworth, London, 1973. 
[6] Z. Dimitrovová, J.N. Varandas, "Critical Velocity of a Load Moving on a Beam with a Sudden Change of Foundation Stiffness: Applications to HighSpeed Trains", Computers \& Structures, in press, available on-line, DOI: 10.1016/j.compstruc.2008.12.005.

[7] Release 11 Documentation for MAPLE, Maplesoft a division of Waterloo Maple, Inc., 2007.

[8] Release R2007a Documentation for MATLAB, The MathWorks, Inc., 2007.

[9] J.A. Cottrell, A. Reali, Y. Bazilevs, T.J.R. Hughes, "Isogeometric analysis of structural vibrations", Computer Methods in Applied Mechanics and Engineering 195, 5257-5296, 2006.

[10] Release 11.0 Documentation for ANSYS, Swanson Analysis Systems IP, Inc., 2007.

[11] L. Frýba, "Vibration of solids and structures under moving loads". 3rd edition, Thomas Telford, London, 1999.

[12] J.E. Akin, M. Mofid, "Numerical solution for response of beams with moving mass", ASCE Journal of Structural Engineering 115(1), 120-31, 1989. 